\title{
Job switch stymies Japan's abduction probe
}

David Cyranoski, Tokyo

A geneticist has landed a plum job at the Tokyo police department, just weeks after his work rekindled a row over the fate of Japanese citizens abducted by North Korea.

But critics are claiming that the transfer of Tomio Yoshii from Teikyo University to head the forensics unit at the Tokyo metropolitan police department is intended to shield him from enquiries about the accuracy of his DNA tests. In a fractious parliamentary exchange with foreign minister Nobutaka Machimura on 30 March, Nobuhiko Suto, a member of the opposition Democrat Party of Japan, suggested that the government had used its influence to plant Yoshii in his new position.

The Japanese government has claimed that Yoshii's DNA analysis proves beyond doubt that cremated remains provided by the North Korean government late last year were from someone other than Megumi Yokota a Japanese woman kidnapped by North Korea in 1977. Japan has been demanding a full account of the fate of Yokota and several others who were allegedly kidnapped.

But in an interview with Nature, Yoshii has conceded that his results could have been the

\section{IMAGE \\ UNAVAILABLE FOR COPYRIGHT REASONS}

the 30 March debate, Suto told Machimura that it was "surprising" that "a civilian without real police training" should suddenly obtain a top position in the police department. "Isn't this just hiding a witness?" Suto asked.

Yoshii's results were taken as conclusive by the government despite contrary reports from the National Research Institute of Police Science in Chiba, which found that no DNA could be gleaned from the remains. "If we are going to take the word of a single academic at a private university over that of this huge research institute, shouldn't we just get rid of

result of contamination (see Nature 433,445 ; 2005). Japanese government officials have disputed the Nature article, claiming that Yoshii says he was misquoted. A documentary film-maker from Australia, a South Korean broadcasting company and other reporters have since sought, without success, to interview Yoshii.

Suto says he wants Yoshii to testify on the matter before parliament's foreign affairs committee. But in Yoshii's new position with the police force, he can only appear if his employer agrees - an arrangement that Suto says is being used as an obstacle. During the institute?"Suto asked Machimura.

Machimura called Suto's scepticism "an insult" and said that the ministry had taken the investigation seriously. "We were not just trying to pull out some predetermined conclusion," he protested. "I wish you would choose your words more carefully."

Suto still plans to call Yoshii to testify, and says he will get to the bottom of why the government set so much store by Yoshii's results. "If Japan keeps going in this direction," he warns, "it will undermine its scientific reputation.”

Additional reporting by Junko Chikatani.

\section{Image problems jeopardize comet mission's impact}

\section{Rex Dalton, San Diego}

Researchers are working against the clock to fix a blurry telescope whose malfunction could undermine a $\$ 330$-million US space mission to a distant comet.

The Deep Impact spacecraft is on course for comet Tempel 1, about 160 million kilometres from Earth. The car-sized craft has an 'impactor' that is due to split off and strike the comet on 4 July, while cameras on the main spacecraft capture the collision, enabling researchers to analyse the comet's make-up in unprecedented detail.

But Deep Impact's main instrument - a high-resolution telescope on the fly-by probe - is out of focus, admit project officials at the Jet Propulsion Laboratory (JPL) in Pasadena, California. This will affect the quality of the images captured from the experiment, the officials say.

The JPL project team has been working quietly for more than a month to try to fix the focus problem, which was discovered shortly after the probe was launched in Florida on 12 January. NASA, which is funding the project, acknowledged the

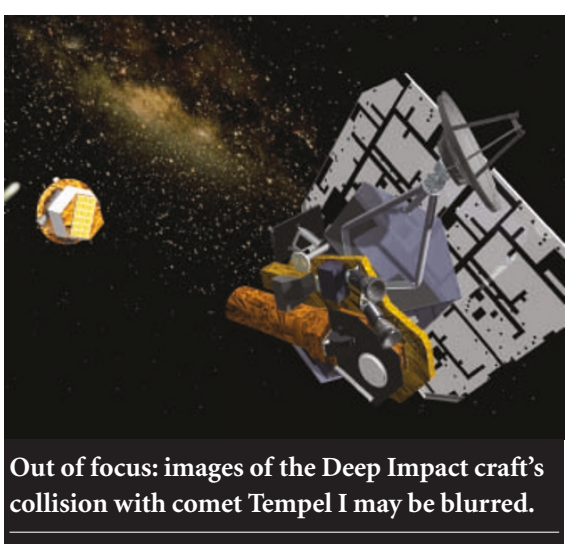

problem on 25 March, after an enquiry from Nature.

JPL scientists have organized a special team to study possible remedies. But so far, efforts to correct the focus have failed, JPL officials say.

Project scientists now plan to employ computer imaging techniques that they hope will improve data from Deep Impact. Similar techniques have been successfully employed in the past on other space telescopes, including Hubble, they say.

The problem telescope was built by Ball Aerospace \& Technologies of Boulder, Colorado. Ball officials refused requests for an interview.

As the Deep Impact spacecraft approaches Tempel 1, the impactor will separate from the main craft. The impactor itself has an onboard telescope and camera, which will be used for navigation and for capturing images up to the point of collision.

The main spacecraft is due to fly past the comet at a distance of about 700 kilometres. It carries the problematic high-resolution telescope, as well as a back-up mediumresolution instrument, which is due to capture a wider view of the collision. The back-up instrument is functioning properly, officials say.

But even if pictures from the highresolution telescope cannot be improved, the instruments are expected to provide "the most detailed pictures of a comet ever taken", says Michael A'Hearn, an astronomer from the University of Maryland at College Park who is chief scientist on the project. 